MRS Advances (C) The Author(s), 2020, published on behalf of Materials Research Society by Cambridge University Press. This is an Open Access article, distributed under the terms of the Creative Commons Attribution licence (http://creativecommons.org/licenses/by/4.0/), which permits unrestricted re-use, distribution, and reproduction in any medium, provided the original work is properly cited.

DOI: 10.1557/adv.2020.346

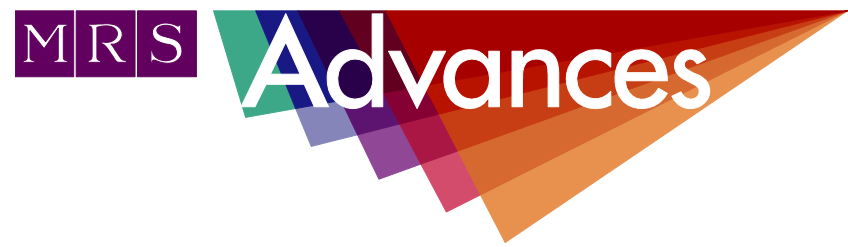

\title{
Quantitative Disorder Analysis and Particle Removal Efficiency of Polypropylene-Based Masks
}

\author{
R.A. Makin ${ }^{1}$, K.R. York ${ }^{1}$, A.S. Messecar ${ }^{1}$, S.M. Durbin ${ }^{1, *}$ \\ ${ }^{I}$ Department of Electrical and Computer Engineering, Western Michigan University, \\ Kalamazoo, MI 49008 USA
}

*Corresponding author:durbin@ieee.org

ABSTRACT

We demonstrate a methodology for predicting particle removal efficiency of polypropylene-based filters used in personal protective equipment, based on quantification of disorder in the context of methyl group orientation as structural motifs in conjunction with an Ising model. The corresponding Bragg-Williams order parameter is extracted through either Raman spectro-scopy or scanning electron microscopy. Temperature-dependent analysis verifies the presence of an order-disorder transition, and the methodology is applied to published data for multiple samples. The result is a method for predicting the particle removal efficiency of filters used in masks based on a materiallevel property.

\section{INTRODUCTION}

There are many applications where the introduction of controlled disorder into a material can enhance a property of interest. The study of quantifying the degree of disorder in materials emerged in the middle $20^{\text {th }}$ century from $\mathrm{x}$-ray diffraction studies of binary metal alloys, such as $\mathrm{AuCu}$ and $\mathrm{ZnCu}$ [1-4]. In order to describe the observed changes in $\mathrm{x}$-ray diffraction peak intensity as samples of metal binary alloys were heated, 
Bragg and Williams defined an order parameter, $S$ [1-3], commonly referred to now as the Bragg-Williams order parameter. For an alloy with constituent elements $A$ and $B$, it may be expressed as

$$
S=r_{A}+r_{B}-1
$$

where $r_{A}$ is the fraction of " $A$ " atoms on $A$-atom lattice sites, and $r_{B}$ is the fraction of " $B$ " atoms on $B$-atom lattice sites. In this context, we reference the perfectly ordered structure where all atoms are on their respective (ideal) site. For a such a sample $S$ is equal to unity, which means that the sample must have: equal numbers of $A$ and $B$ atoms, $A$-atom lattice sites only occupied by $A$ atoms, and $B$-atom lattice sites only occupied by $B$ atoms (i.e. $r_{A}$ $\left.=r_{B}=1\right)$. At the other extreme for such a sample, in which the $A$ and $B$ atoms are randomly distributed over both $A$-atom and $B$-atom lattice sites (i.e. $r_{A}=r_{B}=0.5$ ), $S$ is equal to 0 .

Traditionally, the order parameter has been determined through $\mathrm{x}$-ray diffraction [4]. However, we have recently extended measurements of $S$ to other experimental techniques, including Raman spectroscopy, reflection high-energy electron diffraction, transmission electron microscopy and scanning electron microscopy (SEM) [5-7]. It is prudent to note here that each of these techniques directly measures $S^{2}$ instead of $S$, and thus we report $S^{2}$ values. Further, as we demonstrate, it is possible to correlate disorder to system-level properties in certain situations through the application of an Ising model, where a linear relationship between the property and $S^{2}$ emerges. We have applied this approach to a wide range of materials including heterovalent semiconductors, binary and elemental semiconductors, and even biological systems such as viruses [5-7]. Given the contempo-rary COVID-19 pandemic and the corresponding surge in demand for personal protective equipment, we were motivated to investigate the application of this technique to materials commonly used in masks and filters. Thus, in this report, we provide evidence of the disorder phenomenon in polypropylene-based fibers through analysis of published temperature-dependent measurements. Further, building upon established knowledge of polypropylene tacticity, we propose a corresponding Ising model and demonstrate its applicability to respiratory masks used to mitigate the risk of inhalation of viral particles conveyed through exhaled droplets.

\section{METHODS}

We measured the $S^{2}$ value of published Raman spectra of polypropylene samples based on the work of Loveluck and Sokoloff [8], as well as from intensity analysis of electron microscopy images. Peaks in a Raman spectrum associated with the $S$ $=1$ ordered structure have integrated intensities proportional to $S^{2}$, while peaks associated with the completely disordered structure have an integrated intensity proportional to (1 $S^{2}$ ). The corresponding equations presented by Makin et al. can then be rearranged to extract the order parameter from the Raman spectrum of a single sample [5]. Specifically,

$$
J_{S=1} / J_{S=0}=S^{2} /\left(1-S^{2}\right)
$$

where $J_{S=1}$ is the integrated intensity of a peak associated with the ordered structure, and $J_{S=0}$ is the integrated intensity of the disordered structure feature. Figure 1a shows the results of $S^{2}$ analysis on a published Raman spectrum of a polypropylene sample [9], 
where the peak near $970 \mathrm{~cm}^{-1}$ is the peak associated with disordered polypropylene and the peak near $995 \mathrm{~cm}^{-1}$ is the peak associated with completely ordered polypropylene.

In the case of SEM image analysis, the $S^{2}$ value of a sample is equal to the percentage of sample image area corresponding to bright regions [7]. However, bright and dark areas corresponding to the ordered and disordered regions, respectively, can be more easily identified by first thresholding the image near the average pixel intensity of the bright regions. The analysis process involves fitting two curves to the pixel intensity histogram, one representing the disordered regions and one representing the ordered regions (Figure 1b). The threshold for the image is normally chosen at the peak of the ordered curved, although it may be selected at an integer multiple of the standard deviation, $\sigma$, away from the peak depending on the relative location of the intersection between the ordered and disordered curves. The fitted data can be accessed in the online repository specified in ref. [11].
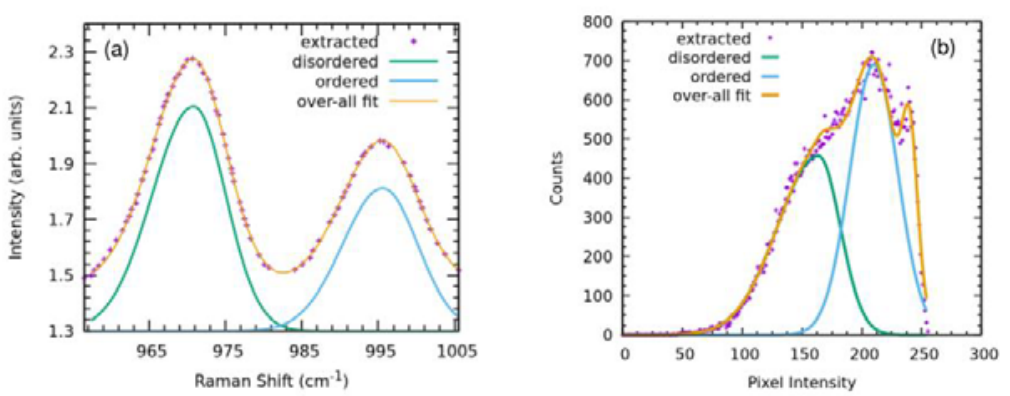

Figure 1. a) Example of Raman analysis of a polypropylene sample, resulting in a value of $S^{2}=0.3937$. Data extracted from ref. [9]. b) Example of $S^{2}$ analysis on a pixel intensity histogram of an SEM image of a different polypropylene sample, resulting in an $S^{2}$ value of 0.5152 . Data extracted from ref. [10].

\section{RESULTS AND DISCUSSION}

One method for experimentally verifying that a material or system can have states of varying degrees of ordering is by measuring $S^{2}$ of a sample as a function of temperature. Landau theory describes order-disorder transitions as second-order transitions [12], and as a result, the order parameter of a system as a function of temperature is

$$
S(T)=\sqrt{\alpha_{0}\left(T_{C}-T\right) / \beta}
$$

where $\alpha_{0}$ and $\beta$ are material-dependent constants, and $T_{C}$ is the critical temperature below which $S=0$. Squaring both sides of Eq. 3 yields

$$
S^{2}(T)=\left(\alpha_{0} / \beta\right) T_{C}-\left(\alpha_{0} / \beta\right) T
$$

Therefore, if $S^{2}$ exhibits a linear trend with temperature for a system, it can be taken as evidence that the system is undergoing an order-disorder transition. 
Figure 2 shows the results of such an analysis applied to the reported Raman spectra of a polypropylene sample systematically heated in $5{ }^{\circ} \mathrm{C}$ increments from $30{ }^{\circ} \mathrm{C}$ to $225^{\circ} \mathrm{C}$ [9]. In order to apply Eq. 2 to extract $S^{2}$, two peaks must be first identified in the Raman spectrum of polypropylene, one arising from disorder, and one arising from order. According to Landau theory, $S$ should decrease with increasing temperature, and thus disorder-related peaks (which have a $\left(1-S^{2}\right)$ dependence) should increase in intensity with increasing temperature, whereas peaks associated with the ordered structure (which have an $S^{2}$ dependence) should decrease in intensity with increasing temperature. From Figure 2a, we can see that with increasing temperature, the peak at $995 \mathrm{~cm}^{-1}$ is decreasing in intensity while the peak at $970 \mathrm{~cm}^{-1}$ is increasing in intensity; thus, the peak at $995 \mathrm{~cm}^{-}$ ${ }^{1}$ is associated with the ordered structure and the peak at $970 \mathrm{~cm}^{-1}$ is associated with the disordered structure. Using these two peaks and Eq. 2, we have extracted $S^{2}$ for the Raman spectra reported by Hiejima et al. for temperatures up to the melting point [9]. The results, plotted as a function of temperature in Figure $2 \mathrm{~b}$, show a clear linear trend between $S^{2}$ and temperature - as predicted by Landau theory for a system undergoing an order-disorder transition. This provides additional evidence that polypropylene polymers can have some degree of disorder somewhere within their structure, as has been previously established using x-ray diffraction [13-15].
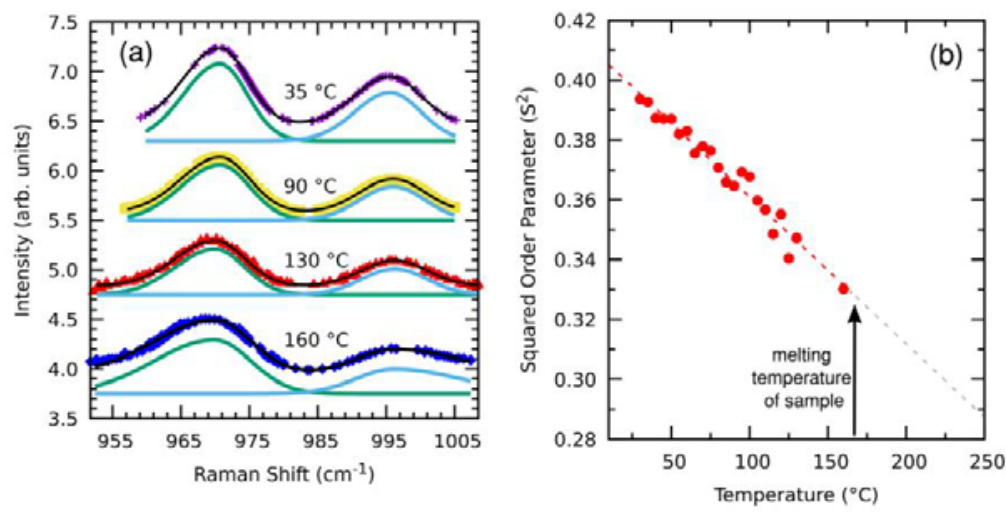

Figure 2. a) Raman spectra for polypropylene from Hiejima et al. [9] with fits to the peaks near 970 $\mathrm{cm}^{-1}$ and $985 \mathrm{~cm}^{-1}$ for selected temperatures. b) $S^{2}$ as a function of temperature for polypropylene extracted from Raman spectra from Hiejima et al. [9]. For all data points in (b) the error bars for $S^{2}$ values are within the size of the symbols.

Another property of polypropylene that can be obtained from the data in Figure $2 \mathrm{a}$ is the critical temperature, $T_{C}$. From Eq. $3, T_{C}$ is equal to the dependent-axis intercept of the line defined by the $S^{2}$ versus $T_{C}$ relationship. Applying linear regression to the data in Figure $2 \mathrm{~b}, T_{C}$ for the order-disorder transition in the polypropylene sample of Hiejima et al. is found to be $834^{\circ} \mathrm{C}$. This transition temperature is well above the melting point of polypropylene, which would seem to rule out the possibility of achieving a zero value of $S^{2}$, or even an $S^{2}$ value below 0.30 . However, it is possible to achieve such $S^{2}$ values through non-equilibrium growth conditions. For example, we have achieved $S^{2}$ close to 0 for $\mathrm{ZnSnN}_{2}$ through plasma-assisted molecular beam epitaxy at a temperature of $420{ }^{\circ} \mathrm{C}$ [5], a non-equilibrium crystal growth technique, despite the fact that the critical temperature for $\mathrm{ZnSnN}_{2}$ is predicted to be over $3000 \mathrm{~K}$ [16]. An example of a technique 
for polypropylene that can reach $S^{2}$ values corresponding to temperatures above its melting point is melt-electrospinning, a process commonly used in mask production. Figure 3 shows the influence of the applied voltage on the $S^{2}$ value of the resulting polypropylene during a melt-electrospinning process [17], confirming that such an approach can be used to access low values of the Bragg-Williams order parameter for this material.

Disorder in polypropylene is related to variations in orientation of the methyl groups relative to the polymer chain. This is commonly referred to as tacticity; the methyl groups are on the same side of the chain for isotactic polypropylene, on alternating sides for syndiotactic polypropylene, and randomly aligned for atactic polypropylene. This tacticity can be represented using an Ising model with a spin "up" is assigned to methyl group on one side of the polymer chain and a spin "down" to a methyl group located on the opposite side of the polymer chain. Similar Ising models have previously been developed and applied to related polymers, such as isotactic vinyl polymer [18-21]. However, our approach is fundamentally different. Whereas previous models considered the entire set of possible sequences that can occur in a given chain, we describe disorder in terms of the percentages of the structural motifs present in the polymer. While our set of complete structural motifs is contained within the previous models, those models obscure the fundamental importance of the variety of structural motifs in determining system-level properties.

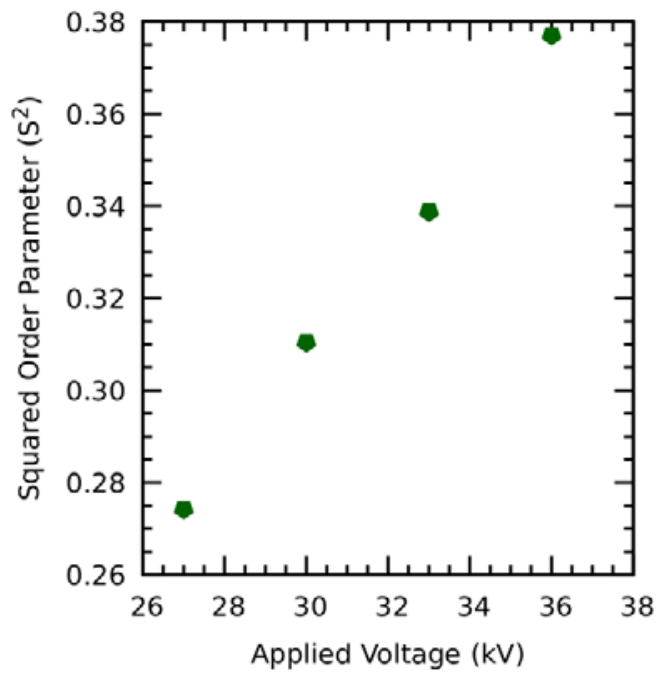

Figure 3. Calculated $S^{2}$ value of polypropylene versus the voltage used during the melt-electrospinning process employed to make the material. Data extracted from ref. [17]. For all data points, the error bars for $S^{2}$ values are within the size of the symbols.

Following our previously reported methodology of modelling disorder by combining structural motif distributions and the Ising model [5-7], we can identify the reference structural motif associated with the ordered structure as three polypropylene blocks with methyl groups on alternating sides with respect to each other, as shown inset 
in Figure 4a. This defines the syndiotactic structure as the $S=1$ structure (since an $S=1$ structure must have equal numbers of spins, which in this case corresponds to equal numbers of methyl groups on opposite sides of the polymer chain). There are six other possible structural motifs, one of which - the $A_{3}(1)$ motif - is shown inset in Figure $4 \mathrm{~b}$. In this notation scheme, A denotes a methyl group oriented in the "upward" direction (for the orientation of the polymer chain shown in the Figure 4 insets) and B denotes a methyl group oriented in opposite direction, which in this case is out of the page as shown by the red and black highlighted methyl groups in the inset of Figure $4 a$ for the $\mathrm{AB}_{2}(0)$ motif. The number in parenthesis represents the number of methyl groups in the opposite orientation of the corresponding methyl group characterizing the reference $(S=1)$ motif. Thus, $\mathrm{A}_{3}(1)$ denotes the motif with all upward-oriented methyl groups, with one methyl group misoriented relative to the reference motif.

The six other possible structural motifs only occur within the polymer when some degree of disorder is present in the structure, with the percent occurrence depending on both $S$ and $x$, where $x$ is the fraction of methyl groups oriented along a specific side of the polymer. Thus, isostatic polypropylene with methyl groups oriented along one side would have $x=1$, while isostatic polypropylene with methyl groups oriented along the opposite side would have $x=0$. Figure 4a provides the percentage of each motif present in polypropylene with $x=0.5$ as a function of $S^{2}$, and Figure $4 \mathrm{~b}$ plots the percentage of each motif at $S=0$ as a function of $x$.
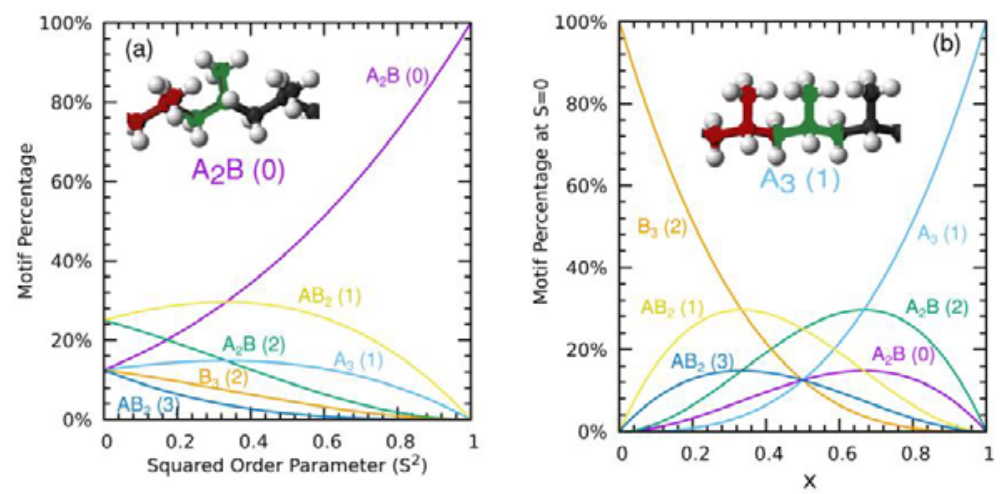

Figure 4. a) The percentage of each structural motif for polypropylene as a function of $S^{2}$ at the balanced, ideal composition of $x=0.5$. b) The percentage of each structural motif for polypropylene as a function of $x$ at $S=0$. Inset are two possible motifs: the reference motif $\mathrm{A}_{2} \mathrm{~B}(0)$, and a $\mathrm{A}_{3}(1)$ motif.

Following the technique used by Zunger and Wei [22, 23], using the spin modeling along with a cluster expansion out to the pair-interaction terms, we have shown [7] that a system-level property dominated by pair interactions can be expressed as

$$
P(x, \mathrm{~S})=[P(x=0.5, \mathrm{~S}=1)-P(x, S=0)] S^{2}-P(x, S=0),
$$

where $P(x, S)$ is the system property at the given composition $x$ and degree of ordering $S$. To investigate whether the particle removal efficiencies of polypropylene masks and filters is such a system-level property of this material, we extracted $S^{2}$ values of polypropylene masks and filters via published SEM images [10] and determined that the reported filtering efficiency (measured using organic particulate matters generated from 
dioctyl phthalate [10]) does indeed follow the linear trend with $S^{2}$ predicted by Eq. 5, as shown in Figure 5a. Interestingly, the efficiency of the filters and masks increases with increasing disorder in the polypropylene. A possible explanation for this trend may be found by considering the structural motifs that occur with increasing disorder. Specifically, the motifs dominated by methyl groups on the same side of the polymer chains occur in increasing percentages as $S$ decreases, as can be seen in Figure 4 a for the case of $x=0.5$, while the percentage of the reference motif with a sequence of methyl groups on alternating sides decreases with decreasing $S^{2}$. This suggests that same-side methyl-group-dominated motifs may provide a more significant barrier to particles passing through than the alternating-side reference motif, leading to better filtering efficiencies for more disordered polypropylene structures.
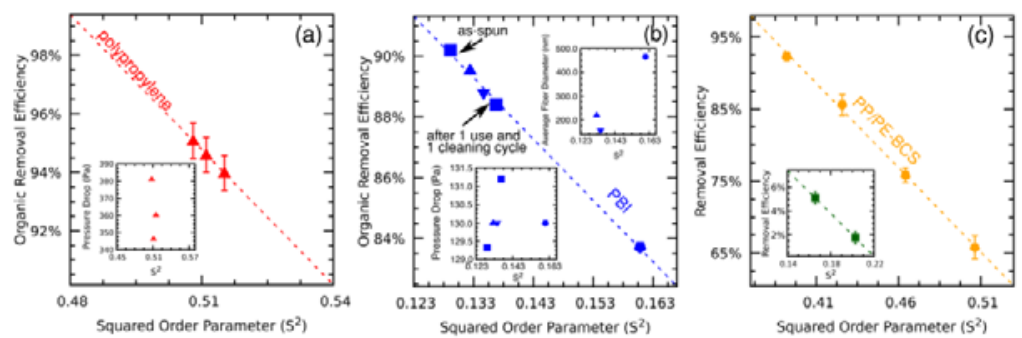

Figure 5. a) Organic removal efficiencies as a function of $S^{2}$ for 3 different commercial polypropylenebased mask filters. Inset is the pressure drop across each filter as a function of $S^{2}$. b) The organic removal efficiencies of polybenzimidazole (PBI) filters as a function of $S^{2}$. Inset at the bottom left is the pressure drop across each filter as a function of $S^{2}$, and inset at the top right is the average fiber diameter as a function of $S^{2}$. All data in (a) and (b) were extracted from ref. [10]. c) Fil-tering efficiencies as a function of $S^{2}$ for polypropylene/ polyethylene bicomponent spunbond (PP/PE- BCS) fibers. Data extracted from ref. [24]. Inset is the filtering efficiency as a function of $S^{2}$ for filters made of polyester. Data extracted from ref. [25]. For all data points in (a), (b) and (c), the error bars for $S^{2}$ values are within the size of the symbols, and error bars for measured quantities from the literature, such as removal efficiencies, are included when reported.

We have also applied $S^{2}$ analysis to masks made of other fibrous materials, such as polybenzimidazole (PBI), polyester and polypropylene/polyethylene bicomponent spunbond fibers (PP/PE-BCS). Figure 5 shows the results of these analysis for these materials. For the PBI samples, the filtering efficiency was measured using organic particulate matters generated from dioctyl phthalate [10]. For the PP/PE-BCS, the removal efficiencies were measured using charge neutral sodium chloride [24], and for the polyester samples the removal efficiencies were measured for particles with a diameter of 0.5 microns [25]. As Figure 5b,c and the inset of Figure 5c illustrate, as in the case of polypropylene, each of these materials demonstrates a linear relationship between their filtering efficiency and $S^{2}$.

While the efficiency of a filter is typically associated with features such as the fiber diameter (with smaller diameter fibers yielding higher removal efficiencies [10]), the trend seen in the removal efficiencies is actually due to the ordering of the material. Evidence for this can be seen in the graph inset in top right of Figure 5b, which shows the 
average fiber diameter as a function of $S^{2}$ for three PBI films. The plot shows that there is no linear trend between the average fiber diameter and $S^{2}$, while for the same three filters there is a linear trend between the $S^{2}$ of the polymers and the filter removal efficiency. Thus, the results shown in Figure 5 provide initial evidence that this model of disorder that we have developed for polypropylene has applications to the broader family of polymer-based fibers.

Additionally, the insets to Figure $5 \mathrm{a}$ and $5 \mathrm{~b}$ plot the pressure drop across the polypropylene and PBI based filters as a function of $S^{2}$. While all of the samples of each material lie on the same $S^{2}$ line for the removal efficiency, the pressure drops of the same filters do not fall along a single line for each material. Thus, there is no linear relationship between $S^{2}$ of the material and the pressure drop of the type predicted by the spin-based model we have developed. This result provides evidence that pressure drop, unlike the filter efficiency, is not dominated by the ordering of the polymers, and instead is driven by the larger structural features of the filter, such as number and density of layers in the filter. As Hiejima et al. [9] and Lee et al. [10] demonstrate, Raman spectroscopy and SEM are straightforward and powerful techniques for characterizing and understanding these fiber structures. Our model presented here, along with those results, provides a potential pathway for achieving masks with better filtering efficiencies and lower pressure drops, by tuning $S^{2}$ of the polymers in the filter and the overall structure of the filter, respectively, using standard and readily available techniques.

\section{CONCLUSIONS}

We have provided evidence for an order-disorder transition in polypropylene based on temperature-dependent measurements of the Bragg-Williams order parameter $S$. A model is proposed for the corresponding structural disorder based on the alignment of methyl groups along the polymer chain. Additionally, a system level property of polypropylene - its particle filtering efficiency - follows the predicted spin (Ising) based model equation relating material properties and $S^{2}$. The results provide a framework for application to other polymers and fiber systems to quantify and understand the impact of structural disorder at the material level in these systems, which can be used to evaluate filter designs, mask cleaning strategies, and potentially even form the basis for quality control in manufacturing.

\section{ACKNOWLEDGEMENT}

This work stems from studies of disorder in $\mathrm{ZnSnN}_{2}$ and $\mathrm{MgSnN}_{2}$, funded in part by the National Science Foundation (grants DMR-1410915 and DMR-2003581), and by a Western Michigan University Faculty Research and Creativity Activities Award.

\section{REFERENCES}

[1] W. L. Bragg and E. J. Williams, Proceedings of the Royal Society of London. Series A, Containing Papers of a Mathematical and Physical Character 145, 699 (1934).

[2] W. L. Bragg and E. J. Williams, Proceedings of the Royal Society of London. Series A - Mathematical and Physical Sciences 151, 540 (1935). 
[3] E. J. Williams and W. L. Bragg, Proceedings of the Royal Society of London. Series A - Mathematical and Physical Sciences 152, 231 (1935).

[4] B. E. Warren, X-Ray Diffraction (Dover Publications, 2012).

[5] R. A. Makin, K. York, S. M. Durbin, N. Senabulya, J. Mathis, R. Clarke, N. Feldberg, P. Miska, C. M. Jones, Z. Deng, L. Williams, E. Kioupakis, and R. J. Reeves, Phys. Rev. Lett. 122, 256403 (2019).

[6] R. A. Makin and S. M. Durbin, bioRxiv:10.1101/2020.06.08.139907 (2020).

[7] R.A. Makin, K.R. York, S.M. Durbin and R.J. Reeves, Phys. Rev. B 102, $115202(2020)$

[8] J. Loveluck and J. Sokoloff, J. Phys. Chem. Solids 34, 869 (1973).

[9] Y. Hiejima, K. Takeda, and K. Nitta, Macromolecules 50, 5867 (2017).

[10] S. Lee, A. R. Cho, D. Park, J. K. Kim, K. S. Han, I.-J. Yoon, M. H. Lee, and J. Nah, ACS Applied Materials \& Interfaces 11, 2750 (2019).

[11] Data and analysis related to measuring the $S^{2}$ for all samples used in this manuscript are available on an online data repository. DOI: 10.5281/zenodo.3904578

[12] In Principles of Equilibrium Statistical Mechanics (John Wiley \& Sons, Ltd, 2005), pp. 432-469.

[13] M. Hikosaka and T. Seto, Polymer Journal 5, 111 (1973).

[14] F. Auriemma, O. Ruiz de Ballesteros, C. De Rosa, and P. Corradini, Macromolecules 33, 8764 (2000).

[15] C. De Rosa, M. Scoti, R. Di Girolamo, O. R. de Ballesteros, F. Auriemma, and A. Malafronte, Polymer Crystallization 3, e10101 (2020).

[16] S. Lany, A. N. Fioretti, P. P. Zawadzki, L. T. Schelhas, E. S. Toberer, A. Zakutayev, and A. C. Tamboli, Phys. Rev. Materials 1, 035401 (2017).

[17] H. Li, W. Wu, M. M. Bubakir, H. Chen, X. Zhong, Z. Liu, Y. Ding, and W. Yang, J. Appl. Polymer Science 131, (2014).

[18] G. Allegra, P. Corradini and P. Ganis, Macromolecular Chemistry and Physics 90, 60 (1966).

[19] T.M. Birshtein and P.M. Luisi, Vysokomol. Soedin. 6, 1238 (1964).

[20] R.-M. Ho, Y.-W. Chiang, S.-C. Lin, and C.-K. Chen, Progress in Polymer Science 36, 376 (2011).

[21] R. Ishidate, A. J. Markvoort, K. Maeda, and E. Yashima, J. Am. Chem. Soc. 141, 7605 (2019).

[22] D. B. Laks, S.-H. Wei, and A. Zunger, Phys. Rev. Lett. 69, 3766 (1992).

[23] S. Wei, D. B. Laks, and A. Zunger, Applied Physics Letters 62, 1937 (1993).

[24] J. Liu, X. Zhang, H. Zhang, L. Zheng, C. Huang, H. Wu, R. Wang, and X. Jin, RSC Advances 7, 43879 (2017).

[25] I. E. Agranovski, R. Huang, O. V. Pyankov, I. S. Altman, and S. A. Grinshpun, Aerosol Science and Technology 40, 963 (2006). 